\title{
SUMÁRIO
}

EDITORIAL

423

\section{IS SUSTAINABILITY IN BUSINESS STRATEGY FACTUAL OR}

FIGURATIVE?

Monique Cristiane de Oliveira, Anastácia Rosa Portella, Suliani Rover, Denize

Demarche Minatti Ferreira, José Alonso Borba

OS CONSÓRCIOS EMPRESARIAIS PORTUGUESES: ANÁLISE

ESTATÍSTICA DA VERTENTE ECONÓMICA

Bruno José Machado de Almeida

COMPETÊNCIAS NA FORMAÇÃO EM ADMINISTRAÇÃO: UM

ESTUDO EM CURSO DE GRADUAÇÃO DE UNIVERSIDADE

PÚBLICA BRASILEIRA

Rogéria Ghedin Servidei Sant'ana, Amyra Moyzes Sarsur, Simone Costa Nunes,

Vera L. Cançado

ANÁLISE DOS CUSTOS AMBIENTAIS RELACIONADOS COM A

GESTÃO DE RESÍDUOS EM INDÚSTRIAS QUÍMICAS

Margareth Aparecida Moraes, Vanessa Theis, Margarete Blume Vier, Dusan Schreiber

REPUTAÇÃO GERA VALOR PARA OS ACIONISTAS? UMA

ANÁLISE NAS EMPRESAS BRASILEIRAS

Alan Diógenes Góis, Márcia Martins Mendes De Luca, Gerlando Augusto Sampaio

Franco de Lima, Alessandra Carvalho de Vasconcelos

CAPITAL SOCIAL E TURISMO RURAL EM UMA ASSOCIAÇÃO DO NORTE DO RIO GRANDE DO SUL: UM ESTUDO DA ROTA DAS

SALAMARIAS.

Jaqueline dos Santos, Fabiana Melara, Eliana Andréa Severo, Janaina Macke

IMPACTO DOS RATINGS DE CRÉDITO NAS AÇÕES DE

EMPRESAS DE CAPITAL ABERTO NO BRASIL

Rafaela Augusta Cunha Silveira, Renata Turola Takamatsu, Bruna Camargos Avelino

OS EFEITOS DOS ROYALTIES DA MINERAÇÃO SOBRE A PROMOÇÃO DO DESENVOLVIMENTO ECONÔMICO DOS MUNICÍPIOS BAIANOS: UMA ANÁLISE DO PERÍODO DE 2009 A 2011 POR MEIO DA ABORDAGEM DEA

Jéssica da Silva Cerqueira, Adriano Alves de Rezende, Carlos Eduardo Ribeiro Santos 
PERFORMANCE E FOCO DO GESTOR EM FUNDOS

MULTIMERCADOS

Patrycia Olivo Moreira, Vitor Borges Tavares, Rodrigo Fernandes Malaquias

REFLEXÕES SOBRE O COMPORTAMENTO ECOLOGICAMENTE

CORRETO DO CONSUMIDOR CAPIXABA 655

Leandro de Athayde Hemerly, Marcia Juliana d'Angelo

COMPETÊNCIAS GERENCIAIS DOS COORDENADORES DE CURSOS DE INSTITUIÇÕES PRIVADAS DE ENSINO SUPERIOR

NA CIDADE DE FORTALEZA, CE. 681

Jesuína Maria Pereira Ferreira, Kely Cesar Martins de Paiva

MAL-ESTAR NAS ORGANIZAÇÕES: POR QUE OS JOVENS ESTÃO

ABANDONANDO O MUNDO CORPORATIVO? .703

Ana Heloisa da Costa Lemos, Mariana Soares Pinto, Marcelo Almeida de Carvalho Silva

DETERMINANTES DA DIVULGAÇÃO DE INFORMAÇÕES DE RISCO DE MERCADO POR EMPRESAS NÃO FINANCEIRAS 729

Benedito Manoel do Nascimento Costa, Paulo Henrique Leal, Vera Maria Rodrigues Ponte

FATORES DE RESISTÊNCIA À IMPLANTAÇÃO DO SISTEMA DE CONHECIMENTO DE TRANSPORTE ELETRÔNICO (CT-E) EM DUAS GRANDES TRANSPORTADORAS RODOVIÁRIAS DE CARGAS...757 Taís Daiane Assumpção Bianchet, Rodney Wernke

IDENTIDADE, ARTE E GESTÃO EM PROL DO

EMPREENDEDORISMO CULTURAL: SARAU EMPREENDEDOR COMO TECNOLOGIA SOCIAL .783

Israel Marques Campos, Eduardo Davel

FOCO E ESCOPO .809

MISSION AND FOCUS 809

DIRETRIZES PARA AUTORES .811 